\title{
Strain-dependent sexual dimorphism in the extinction of conditioned taste aversion in mice
}

\author{
DONALD K. INGRAM and TIMOTHY P. CORFMAN \\ The Jackson Laboratory, Bar Harbor, Maine 04609
}

\begin{abstract}
Sucrose aversions were induced by lithium chloride toxicosis in 9-week-old male and female mice of the C57BL/6J and DBA/2J strains. Under conditions of ad-lib access to fluids which required a long interval between the sucrose and toxicosis, the conditioned aversions were relatively weak when compared with the sucrose consumption of saline-injected controls. The aversions extinguished rapidly within all groups. Contrary to earlier reports in rats, no sexual dimorphism in extinction rate was observed in this paradigm. When a fluid-deprivation schedule was implemented in the conditioning procedure to shorten the interval between taste experience and toxicosis, strong aversions were noted; yet relatively fast extinction under ad-lib conditions occurred in all groups except male DBA/2J mice. No sexual dimorphism was observed in mice of the C57BL/6J genotype, even when two pairings between sucrose and toxicosis were administered.
\end{abstract}

Chambers and Sengstake (1976) reported sexual dimorphism in the rate at which rats extinguish aversions to sucrose solutions induced by lithium chloride $(\mathrm{LiCl})$ toxicosis. Using this conditioned taste aversion (CTA) paradigm, systematic research by Chambers (1976) provided evidence of the involvement of testosterone in this learning phenomenon. Specifically, mature male rats exhibit much stronger resistance to extinction relative to that reported for mature female rats, but the extinction pattern of castrated male rats parallels that of female counterparts, both intact and castrated. When castrated males and females are provided testosterone propionate replacement, the course of CTA extinction is similar to that of intact males. Replication of this work investigated possible estrogenic and androgenic components of the testosterone effect (Earley \& Leonard, 1978). Androgens, but not estrogens, were found to mediate the slower rate of CTA extinction observed in male rats.

Further investigation demonstrated that the phe-

This study was supported in part by a research grant, AG-00250, awarded to R. L. Sprott, and by General Funds of the Jackson Laboratory. The authors wish to acknowledge Eunice L. Corfman for her valuable editorial assistance. This research was conducted at the Jackson Laboratory while D. K. Ingram was a Postdoctoral Associate supported by a training grant, $\mathrm{MH}-12126$, and a postdoctoral fellowship, AG-05171. Reprint requests should be submitted to Donald K. Ingram, Staff Fellow, Gerontology Research Center, National Institute on Aging, Baltimore City Hospitals, Baltimore, Maryland 21224. T. P. Corfman was a participant in the 1979 Summer College Student Program of the Jackson Laboratory. His present address is the Department of Physiology, Rush University, Chicago, Illinois 60612. The Jackson Laboratory is fully accredited by the American Association for Accreditation of Laboratory Animal Care. nomenon was subject to environmental manipulation. Sexual dimorphism is most reliably observed when fluids are provided ad lib during the extinction process (Chambers \& Sengstake, 1976; Sengstake \& Chambers, 1979; Sengstake, Chambers, \& Thrower, 1978). Fluid deprivation results in decreased resistance to CTA extinction, presumably due to decreased levels of circulating testosterone. Social isolation of males for prolonged periods also results in decreased resistance to extinction, again, presumably related to the effects of testosterone (Chambers \& Sengstake, 1978).

Robbins (Note 1) has extended the observation of sexual dimorphism in the CTA paradigm to another taxon, deer mice (Peromyscus maniculatus bairdi). Consistent with findings in rats, no difference between male and female deer mice was noted in the extinction of a $\mathrm{LiCl}$-induced sucrose aversion when a fluid-deprivation schedule was used during the single-choice testing procedure. When a two-choice procedure was used, the predicted sexual dimorphic pattern emerged. Contrary to the earlier reports in rats, however, Robbins argued that male deer mice exhibited stronger initial acquisition of the CTA than did female deer mice, which would account for their greater resistance to extinction.

These investigations have suggested the involvement of genotype in CTA learning. Thus far, the observations argue for an activational, as opposed to an organizational, role for the hormonal effect (Chambers, 1976). Strain differences in the CTA paradigm have been reported previously for male rats by Dragoin (1971), but the evidence was questioned by Ader (1973) in a replication, the results 
of which suggested that environment might be a more important factor than genotype. The strain differences which Dragoin observed in a cyclophosphamide-induced aversion to hydrochloric acid were between Sprague-Dawley and Long-Evans rats obtained from different suppliers. Ader reported this finding, but found no differences between these two strains obtained from the same supplier.

Strain differences in the CTA paradigm have been reported for inbred male mice (Mus musculus) when ethanol or acetaldehyde has been used as the toxic agent (Dudek \& Fuller, 1978; Horowitz \& Whitney, 1975). These studies observed that ethanol-preferring strains, for example, C57BL/6J (B6) mice, exhibit much weaker aversions to sapid solutions in comparison to ethanol-avoiding strains, for example, $\mathrm{DBA} / 2 \mathrm{~J}$ (D2) mice, when ethanol or acetaldehyde was injected ip. No strain difference between male B6 and D2 mice has been observed in CTA when $\mathrm{LiCl}$ was the toxic agent (Belknap, Belknap, Berg, \& Coleman, 1977; Belknap, Coleman, Foster, 1978; Dudek \& Fuller, 1978). After using a stronger dose of $\mathrm{LiCl}$ than had been used in these previous studies, Ingram (Note 2) observed no evidence of a strain difference in CTA acquisition but found clear evidence for a strain difference in CTA extinction. Male D2 mice exhibited stronger resistance to extinction compared with male B6 mice in one- and two-bottle tests.

These findings suggest the existence of a generalized strain difference in the CTA paradigm that might have genetic components. The present investigation sought to expand the findings of the previous. studies reporting sexual dimorphism in the extinction of CTA to another rodent species and to examine the possible interaction of the dimorphism with genotype.

\section{GENERAL METHOD}

\section{Subjects}

Subjects from the C57BL/6J and DBA/2J strains of house mice (Mus musculus) were obtained from the Division of Animal Resources of the Jackson Laboratory. The animals were placed in standard plastic cages containing wood shavings for bedding and housed in a vivarium maintained at a temperature of $22^{\circ} \pm 2^{\circ} \mathrm{C}$. Music from a radio tuned to an AM station played $18 \mathrm{~h} /$ day to mask background sounds. Lights turned on at 0630 and off at 1830 EST. Throughout all experiments, the mice were provided ad-lib access to Old Guilford $96 \mathrm{~W}$ mouse chow.

\section{Apparatus}

Access to fluids was provided via 50-cc plastic syringes outfitted with curved stainless steel sipper tubes inserted through the holes in the wire tops of the cages. The amount of fluid imbibed was determined at the beginning and end of specified drinking periods by weighing the tubes to the nearest $.1 \mathrm{~g}$. This drinking tube system proved highly efficient with fluid loss due to spillage and/or evaporation estimated to be less than $1 \mathrm{~g}$ per test period.

The CS for all experiments was $15 \%$ sucrose. The sucrose solution was mixed in distilled water $w / v$ and refrigerated about $23 \mathrm{~h}$ before its use. The US for all experiments was a $6.0-\mathrm{mEq} / \mathrm{kg}$ dose of $\mathrm{LiCl}(2 \% \mathrm{bw} .30 \mathrm{M} \mathrm{LiCl})$ injected intraperitoneally (ip). Ingram
(Note 2) had found this dose to be most effective in producing profound taste aversions in the strains of mice studied. The $\mathrm{LiCl}$ injectant was mixed in distilled water $w / v$ on the day of its use and administered with 1 -cc plastic syringes and 26 -ga $3 / 8$-in. hypodermic needles.

\section{EXPERIMENT 1}

This experiment was designed to follow the protocol of previous studies which induced CTA under nondeprived conditions (Chambers, 1976; Chambers \& Sengstake, 1976, 1978; Sengstake \& Chambers, 1979). A comparison of resistance to extinction was made between two genotypes of mice of both sexes.

\section{Method}

From both the B6 and D2 inbred strains, totals of 12 male and 12 female mice were obtained at 6 weeks of age and housed 5 to a cage. At 7 weeks, the mice were housed individually in the standard cages. At 8 weeks, a routine was instituted for measuring water consumption during the first $2 \mathrm{~h}$ after light offset. On Days 1-6, the drinking tubes were removed about $1 \mathrm{~h}$ before light offset, the contents of distilled water were replenished, and the tubes were weighed. At light offset, all animals were handled briefly and the tubes were placed on the cages. After a 2-h period, the tubes were removed, weighed, and replaced on their respective cages.

Conditioning occurred on Day 7 when the animals were approximately 9 weeks old. The mice were provided the CS, a tube of $15 \%$ sucrose, during the 2 -h period after light offset. Following removal of the tubes of sucrose, tubes containing water were placed on the cages. Each animal was weighed, and then half the males and half the females from each strain were administered the US, a $6.0-\mathrm{mEq} / \mathrm{kg}$ dose of $\mathrm{LiCl}$. The remaining animals received a saline placebo injection $(2 \%$ bw. $15 \mathrm{M} \mathrm{NaCl}$ ip). Assignment to these respective experimental and control groups was made randomly within each sex-strain group. The weighing and injection schedule was alternated between the treatment conditions ( $\mathrm{LiCl}$ vs. $\mathrm{NaCl}$ ) and was balanced across sex and strain groups. The injection schedule ran from 5 to $45 \mathrm{~min}$ after removal of the sucrose.

During recovery from toxicosis, water intake was monitored on Days 8 and 9 as it had been done on Days 1-6. The first test for acquisition of CTA was conducted on Day 10. Each mouse was provided 2-h access to a tube of sucrose following light offset, in a procedure identical to that on Day 7 . This procedure was repeated on Days 11-14, at the end of which extinction was complete for nearly all animals. Criterion to extinction was defined as the number of days each individual required to return to the level of sucrose consumption observed on Day 7.

\section{Results}

As shown in Table 1, mice that had received the $\mathrm{LiCl}$ injection tended to drink less sucrose during the 2 -h acquisition test on Day 10 than did their counterparts receiving the $\mathrm{NaCl}$ injection. The results of a 2 (strain) by 2 (sex) by 2 (treatment) ANOVA revealed a significant main effect due to treatment $[F(1,40)=18.39, p<.0005]$. However, the analysis did not yield significant main effects due to strain or to sex $(p>.05)$. Instead, there was a significant Strain by Sex interaction $[F(1,40)=4.68, p<.05]$. Tests of the simple main effects (Winer, 1971) indicated that when treatment was ignored, B6 males 
Table 1

Mean Sucrose Consumption (in Grams) During Acquisition Test in Experiment 1

\begin{tabular}{|c|c|c|c|c|}
\hline \multirow{3}{*}{$\begin{array}{l}\text { Treat- } \\
\text { ment }\end{array}$} & \multicolumn{4}{|c|}{ Strain } \\
\hline & \multicolumn{2}{|c|}{$\mathrm{C} 57 \mathrm{BL} / 6 \mathrm{~J}$} & \multicolumn{2}{|c|}{$\mathrm{DBA} / 2 \mathrm{~J}$} \\
\hline & Mean & SD & Mean & SD \\
\hline & \multicolumn{4}{|c|}{ Males } \\
\hline $\mathrm{NaCl}$ & 1.52 & .34 & 1.37 & .21 \\
\hline \multirow[t]{2}{*}{$\mathrm{LiCl}$} & 1.13 & .32 & .65 & .37 \\
\hline & \multicolumn{4}{|c|}{ Females } \\
\hline $\mathrm{NaCl}$ & 1.00 & .39 & 1.43 & .58 \\
\hline $\mathrm{LiCl}$ & .88 & .40 & .77 & .34 \\
\hline
\end{tabular}

Table 2

Mean Sucrose Consumption (in Grams) of Control Groups in Experiment 1

\begin{tabular}{lcccccc}
\hline & \multicolumn{7}{c}{ Sex } & 10 & 11 & 12 & 13 & 14 & Overall \\
\cline { 2 - 7 } & & \multicolumn{5}{c}{ C57BL/6J } \\
Male & 1.52 & 1.68 & 1.77 & 1.75 & 1.77 & 1.69 \\
Female & 1.00 & 1.25 & 1.32 & 1.93 & 1.63 & 1.43 \\
& & \multicolumn{5}{c}{$\mathrm{DBA} / 2 \mathrm{~J}$} \\
Male & 1.37 & 1.65 & 1.70 & 1.95 & 1.65 & 1.66 \\
Female & 1.43 & 1.27 & 1.33 & 2.10 & 1.90 & 1.61 \\
\hline
\end{tabular}

tended to consume more sucrose than B6 females consumed $[\mathrm{F}(1,40)=5.99, \mathrm{p}<.05]$ and more than D2 males consumed $[F(1,40)=4.12, p<.05]$.

Although there was evidence of a conditioned sucrose aversion, its strength was relatively weak compared with previous reports in these mice (Belknap et al., 1977; Belknap et al., 1978; Dudek \& Fuller, 1978; Ingram, Note 2). As further evidence of this weakness, the aversions extinguished rapidly. The mean (and standard deviation) of days to the extinction criterion was $1.8(1.33)$ for $\mathrm{B} 6$ males and 1.3 (.52 days for B6 females, compared with 3.0 (2.00) days for D2 males and $2.5(1.22)$ days for D2 females. Although it appeared that D2 mice took slightly longer to extinguish their aversions, a 2 (strain) by 2 (sex) ANOVA of these data did not uncover significant effects due to strain or to sex or to the interaction (ps $>.05$ ).

Sucrose consumption of the control groups was also analyzed to determine the existence of strain and sex differences. As shown in Table 2, there was little indication of systematic differences in mean sucrose consumption among the various groups accross 5 days of testing. These data were analyzed in a 2 (strain) by 2 (sex) by 5 (days) ANOVA with repeated measures on the last factor. The main effects of strain and sex were not significant (ps >.05). There was a significant main effect due to days $[F(4,100=12.04, p<.0001]$, which indicated a general increase in consumption across days. In addi- tion, there was a significant Sex by Day interaction $[F(4,100)=3.74, p<.01]$. Tests of simple main effects using a conservative estimate of $F$ (Winer, 1971) indicated that females drank less sucrose than males did only on Day $11[\mathrm{~F}(1,20)=9.20, \mathrm{p}<.01]$ and on Day $12[\mathrm{~F}(1,20)=9.44, \mathrm{p}<.01]$.

In addition, there were no differences noted in fluid consumption prior to conditioning. An ANOVA of data on water consumption on Day 6 revealed no significant main effects due to sex or to age or to their interaction ( $\mathrm{ps}>.05$ ) either during the 2-h drinking period following light offset or during the other 22 -h period. Similarly, there were no significant differences in sucrose consumption on conditioning day $(\mathrm{ps}>.05)$.

Differences in fluid consumption did not take into account differences in body weight. As recorded on Day 7, the mean (SD) weights were 24.8 (1.71) $\mathrm{g}$ for B6 males vs. $20.1(1.80) \mathrm{g}$ for B6 females and $22.7(1.16) \mathrm{g}$ for $\mathrm{D} 2$ males vs. $18.9(0.71) \mathrm{g}$ for $\mathrm{D} 2$ females.

\section{Discussion}

The sexual dimorphism in CTA extinction observed in previous studies of rats was not observed in the present experiment using inbred mice. Comparing sucrose consumption between experimental and control groups, it was evident that CTA was induced, but the initial level of aversion was very weak relative to that previously observed when a $6.0-\mathrm{mEq} / \mathrm{kg}$ dose was administered in a deprivation paradigm (Ingram, Note 2). Moreover, in the current experiment, there was rapid extinction of the aversion in all groups. Possibly the weakness of the aversion was due to the long CS-US interval. Strong aversions occur in rats with this delay in toxicosis, although there is a diminution in response when the CS-US interval is lengthened beyond $15 \mathrm{~min}$ (Nachman, 1970). There might be a species difference in the induction of CTA across such long intervals.

\section{EXPERIMENT 2}

This experiment was designed to shorten the CS-US interval. The protocol involved a fluid-deprivation schedule to insure that the animals would consume adequate quantities of the CS during a relatively short period and, thus, produce a situation in which the US injection would be more contiguous to this experience. Although acquisition of CTA would occur under fluid-deprived conditions, the protocol allowed the extinction phase of the experiment to be conducted under nondeprived conditions. Sengstake and Chambers (1979) had observed that a similar paradigm was as effective in producing the sexual dimorphism in extinction rate as was a paradigm in which both acquisition and extinction were conducted under nondeprived conditions. 


\section{Method}

At 7 weeks, 10 male and 10 female B6 mice and 10 male and 10 female D2 mice were obtained and group housed, 5 to a cage, in the vivarium until they were about 8 weeks old. At that time, they were housed individually and given water ad lib. A fluiddeprivation schedule was initiated $24 \mathrm{~h}$ later. On Days 1-4, all animals were provided 10 -min daily access to distilled water during an individually assigned drinking period occurring during the light cycle sometime between 1430 and $1630 \mathrm{~h}$.

Conditioning occurred when the mice were approximately 9 weeks old. On Day 5, the CS, a $15 \%$ sucrose solution, was presented to all animals during the 10 -min drinking period. About 3-8 min following the removal of the tubes, each mouse was weighed and administered the US, a $6.0-\mathrm{mEq} / \mathrm{kg}$ dose of $\mathrm{LiCl}$. The injection schedule was balanced across sex and strain groups. Placebo injection controls were not deemed necessary because the results of Experiment 1 indicated a lack of systematic differences among control groups in sucrose consumption.

On Day 6, all mice were given 10-min access to water. Then, on Day 7, a test for acquisition of a CTA was conducted by providing simultaneous access to a tube of distilled water and a tube of $15 \%$ sucrose. To circumvent the development of position preference, the positions of the two tubes were switched after about $5 \mathrm{~min}$. Then, after $10 \mathrm{~min}$, the tubes were removed and weighed, and preference scores were computed. Sucrose preference scores were calculated as the percentage of sucrose consumption in grams in relation to total fluid consumption in grams.

An extinction period followed immediately in which each animal was given continuous access to a tube of water and a tube of $15 \%$ sucrose. The tubes were weighed daily, and the positions of the tubes, switched. On alternate days, the contents of the tubes were flushed, and the supply of fluids was replenished. Resistance to extinction was measured as the number of days each animal maintained a sucrose preference score below $50 \%$.

\section{Results}

A single treatment with $\mathrm{LiCl}$ in this paradigm produced profound aversion to sucrose, as measured by the preference scores calculated for the 10-min acquisition test on Day 7. The mean (SD) scores were $9.0 \%$ (10.4) for B6 females, $23.0 \%$ (29.3) for B6 males, $10 \%(8.9)$ for D2 females, and $8.0 \%(6.0)$ for D2 males. According to the results of a 2 (strain) by 2 (sex) ANOVA, there were no significant differences in these scores attributable to either strain or sex or to their interaction ( $\mathrm{ps}>.05$ ). Furthermore, using the analysis recommended by Robbins (Note 1), there was no evidence that any of the groups had floored aversions, that is, all means were significantly above zero $(p<.05)$, according to one-tailed $t$ tests. Thus, extinction began from an equivalent, strong level of aversion for all groups.

The mean sucrose preference scores during extinction are presented in Figure 1. A marked sexual dimorphism is observed in the D2 strain only. The mean (SD) days to extinction criterion were $2.8(.63)$ days for B6 females, 1.6 (.67) days for B6 males, 4.1 (2.47) days for D2 females, and 10.5 (5.04) days for D2 males. Because of the marked heterogeneity of variance among these data, a square-root transformation was performed. The transformed data were submitted to a 2 (strain) by 2 (sex) ANOVA. The results of this analysis yielded significant main effects due to strain $[F(1,36)=36.22, p<.0001]$, to

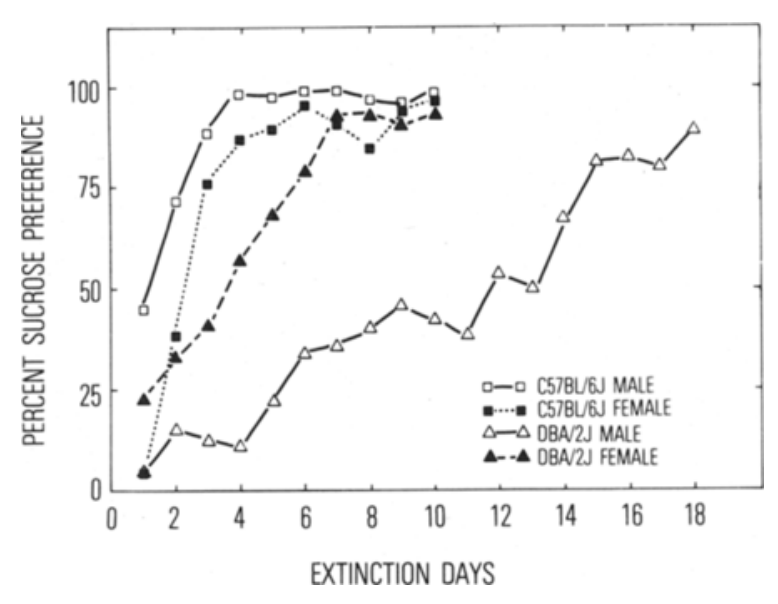

Figure 1. Mean percent sucrose preference during extinction period in Experiment 2.

$\operatorname{sex}[F(1,36)=4.74, p<.05]$, and to the interaction $[F(1,36)=20.55, p<.0001]$. Tests of the simple main effects revealed that the strain difference was significant between male mice $[F(1,36)-55.66, p<$ $.0001]$, and that the sex difference was significant in the $\mathrm{D} 2$ genotype $[\mathrm{F}(1,36)=22.51, \mathrm{p}<.0001]$.

Again there was no evidence of strain differences in thirst motivation between male mice of these genotypes. The mean (SD) water consumption on Day 4 was $1.45(.26) \mathrm{g}$ for B6 males vs. $1.46(.26) \mathrm{g}$ recorded for D2 males. There was a difference in mean (SD) water consumption between the female genotypes, $1.13(.21) \mathrm{g}$ for B6 females vs. $1.55(.15) \mathrm{g}$ for D2 females. A significant Strain by Sex interaction was determined by a 2 (strain) by 2 (sex) ANOVA of these data $[F(1,36)=8.34, p<.01]$. Tests of simple main effects supported the observation that the genotype effect was significant only for females $[F(1,36)=$ $17.49, \mathrm{p}<.0005]$, and that the sex effect was significant only for the B6 genotype $[\mathrm{F}(1,36)=10.15, \mathrm{p}<$ $.005]$. This exact pattern was observed for sucrose consumption on conditioning day. The mean (SD) sucrose consumption on Day 5 was $1.81(.36) \mathrm{g}$ for B6 males vs. $1.85(.21) \mathrm{g}$ for D2 males, and 1.41 (.27) $\mathrm{g}$ for B6 females vs. $1.92(.20) \mathrm{g}$ for $\mathrm{D} 2$ females. A similar analysis of these data revealed the significant Strain by Sex interaction $[F(1,36=7.59, \mathrm{p}<.01]$. Tests of simple main effects again showed the effect of genotype to be significant only for females $[F(1,36)=17.88, p<.0005]$ and the effect of sex to be significant only for the $\mathrm{B} 6$ genotype $[\mathrm{F}(1,36)=11.0$, $\mathrm{p}<.005]$. In summary, there was no evidence that any group exhibited excess thirst motivation relative to the others that might have contributed to a weaker CTA. The only significant difference in thirst motivation was recorded as less fluid intake by B6 females.

Again, the analysis of fluid consumption disregarded differences in body weight, which showed the typical sexual dimorphism. The mean (SD) body 
weight on Day 5 was 17.4 (1.29) $\mathrm{g}$ for B6 females vs. $23.6(2.26) \mathrm{g}$ for $\mathrm{B} 6$ males and $18.3(1.20) \mathrm{g}$ for $\mathrm{D} 2$ females vs. $24.3(2.29) \mathrm{g}$ for $\mathrm{D} 2$ males.

\section{Discussion}

In spite of the acquisition of a profound aversion as measured in the initial 10 -min test, extinction of CTA progressed quickly for all groups except the D2 males. Consistent with an earlier observation (Ingram, Note 2), D2 males exhibited much stronger resistance to extinction in this CTA paradigm than did B6 males. Sexual dimorphism in resistance to extinction was evident only in the D2 genotype, with males requiring over twice as many days to reach criterion. The extinction pattern of B6 males and females was essentially identical.

This Strain by Sex interaction presents some interesting explanations. The testosterone hypothesis of Chambers and her colleagues possibly fits these data according to a previous report on hormone levels in these strains by Eleftheriou and Lucas (1974). They observed that 2-month-old male D2 mice had plasma testosterone levels about $250 \%$ higher than those observed in male B6 mice of the same age. This difference might account for the strain difference between male mice, but there might be other explanations of the failure to note a sex difference in the B6 genotype. One plausible hypothesis concerns the methodology used. The B6 mice extinguished rapidly in this paradigm by requiring only about 2 days to reach criterion. Thus, it was possible that the "window" through which the sexual dimorphism in rate could be observed was too narrow in this paradigm.

\section{EXPERIMENT 3}

As a test of the hypothesis mentioned above, this experiment was conducted in order to lengthen the time required for CTA extinction among B6 mice and thus increase the possibility of observing sexual dimorphism in the extinction pattern. Specifically, the aversion was strengthened by giving two CS-US pairings.

\footnotetext{
Method

Around 7 weeks of age, 10 male and 10 female B6 mice were obtained and group housed, 5 to a cage. At 8 weeks of age, the mice were housed individually, and the fluid deprivation schedule was initiated $24 \mathrm{~h}$ later. The procedure for Days 1-6 was identical to that followed in Experiment 2. Then, on Day 7, a second conditioning trial was conducted. All mice were given access to a tube of sucrose for $10 \mathrm{~min}$ and were then weighed and injected with a $6.0-\mathrm{mEq} / \mathrm{kg}$ dose of $\mathrm{LiCl}$. The schedule of injections was balanced across sex groups. On Day 8 , all mice were provided 10-min access to water. Then, on Day 9, a 10-min preference test between water and sucrose was conducted as it had been done on Day 7 in Experiment 2. Following this test, continuous access to the tubes was provided for 10 days. The sucrose supply for each mouse was replenished every other day, and the positions of the tubes were switched every day. The extinction criterion was a $50 \%$ level of sucrose preference.
}

\section{Results and Discussion}

Male and female B6 mice did not differ significantly on any measure of fluid consumption in this experiment. Table 3 contains the mean estimates of water consumption on Day 4 and of sucrose consumption on Days 5 and 7 . According to the results of individual two-tailed $t$ tests, there were no significant sex differences ( $p s>.05$ ). The data are presented in terms of absolute levels of consumption, which again ignores the differential in mean (SD) body weight, 16.3 (1.57) $\mathrm{g}$ for females vs. $21.7(4.14) \mathrm{g}$ for males.

Following the second CS-US pairing, there was profound aversion in both sexes, as measured in the 10 -min preference test. The mean (SD) sucrose preference score was $4.9 \%$ (8.96) for B6 females vs. 3.5\% (3.75) for B6 males, a difference that was not significant $(t<1.0)$. In contrast with the lack of a statistically significant difference in mean scores between the groups, the results of one-tailed t tests revealed that the mean score for males was significantly above zero $[t(9)=2.94, p<.01]$, whereas the mean score for females was not $[t(9)=1.76, p>.05]$. According to Robbins (Note 1), this analysis suggests that the groups were not equal in strength of conditioning at the beginning of extinction, which makes comparison difficult. Although this statistical argument has merit, it should be noted that the analysis is sensitive to the effects of outliers in the sample. Specifically, when one relatively high score $(\mathbf{2 8 . 6 \%})$ in the female group was removed from the analysis, the mean of 2.4 was then found to be significantly above zero $[\mathrm{t}(8)=1.98, \mathrm{p}<.05]$. Thus, it can be assumed that the two groups were generally equal in strength of conditioning when extinction testing began.

There was no evidence of sexual dimorphism in extinction (see Figure 2). The mean (SD) days to criterion was 6.0 (3.16) days for B6 females compared with 6.4 (2.27) days for B6 males, a difference that was not significant $(\mathrm{t}<1.0)$.

Thus, the multiple conditioning trial procedure was successful in lengthening the time to extinction in B6 mice. In spite of the expanded extinction period, there was no evidence that B6 males showed greater resistance to extinction than $\mathrm{B} 6$ females did.

Table 3

Sex Differences in Mean Fluid Consumption (in Grams) of $\mathrm{C57BL} / 6 \mathrm{~J}$ Mice in Experiment 3

\begin{tabular}{|c|c|c|c|c|}
\hline \multirow[b]{3}{*}{ Day } & \multicolumn{4}{|c|}{ Consumption } \\
\hline & \multicolumn{2}{|c|}{ Female } & \multicolumn{2}{|c|}{ Male } \\
\hline & Mean & SD & Mean & SD \\
\hline & & & & \\
\hline \multirow[t]{2}{*}{4} & 1.32 & .36 & 1.47 & .39 \\
\hline & \multicolumn{4}{|c|}{ Sucrose } \\
\hline 5 & 1.34 & .25 & 1.61 & .37 \\
\hline 7 & .49 & .23 & .48 & .21 \\
\hline
\end{tabular}




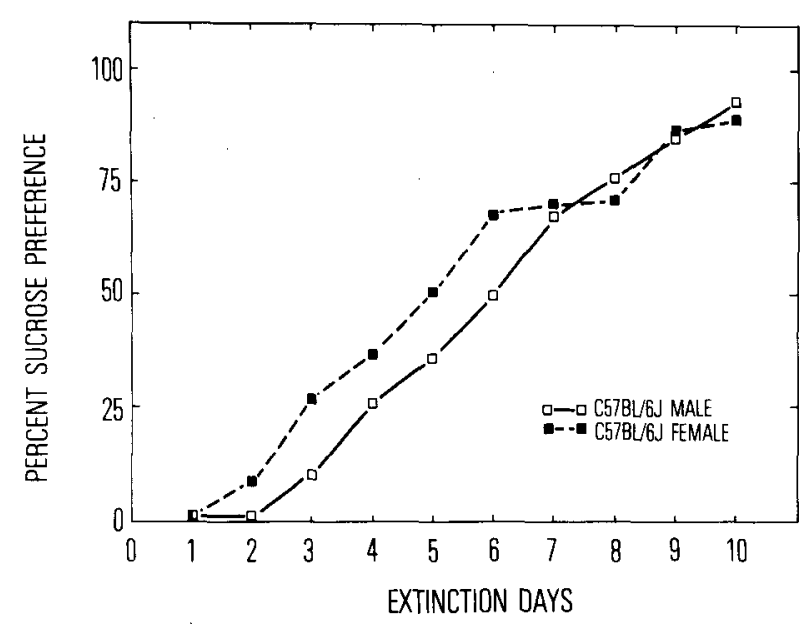

Figure 2. Mean percent sucrose preference during extinction period in Experiment 3.

\section{GENERAL DISCUSSION}

Two replications of previous work were accomplished in the present study. First, the present findings replicated an earlier report that male D2 mice exhibit stronger resistance to extinction of CTA when compared with male B6 mice (Ingram, Note 2). Second, the present results replicated in house mice previous reports that rats and deer mice exhibit sexual dimorphism in the CTA paradigm (Chambers, 1976; Chambers \& Sengstake, 1976; Robbins, Note 1). In support of the findings in the rat studies, sex affected the extinction of CTA rather than the acquisition of CTA, as was suggested in an earlier study of deer mice (Robbins, Note 1). It was necessary, however, to depart from the paradigms used in the earlier studies and to implement a fluid-deprivation schedule in order to induce profound aversions. Moreover, the observation of sexual dimorphism was confined to the D2 strain, in which male mice exhibited stronger resistance to extinction than did females. Male and female mice of the B6 genotype did not differ significantly in extinction rate of a profound CTA following one and even two CS-US pairings.

The observed Strain by Sex interaction is consistent with evidence indicating the involvement of testosterone in determining the rate of CTA extinction (Chambers, 1976; Earley \& Leonard, 1978). Specifically, male D2 mice are reported to have much higher levels $(250 \%)$ of circulating testosterone when compared with male B6 mice at approximately 2 months of age (Eleftheriou \& Lucas, 1974) and, correspondingly, show greater resistance to extinction.

Other factors which might have contributed to the observed differences include differential preference for the CS and differential response to the US. Under ad-lib conditions, B6 mice have been reported to consume greater quantities of sucrose solutions
(Ramirez \& Fuller, 1976). Little evidence was found in the present study to confirm this finding. There was no strain difference in CS consumption in Experiment 1 to indicate greater preference by a particular group which would be correlated with faster extinction. Females in the control groups of both genotypes were observed to consume less sucrose than males did on only 2 days. In Experiment 2, female B6 mice were observed to consume less sucrose on conditioning day than did B6 males, but this difference was not observed in Experiment 3. The question of differential effects of the US is not obvious when based exclusively on the results of the behavioral test. Although extinction began from an equivalent level for all groups in Experiments 2 and 3, it is possible that the degree of toxicosis was more severe in one or more of the groups than in the others, as was the case of ethanol and acetaldehyde toxicity in this paradigm (Dudek \& Fuller, 1978; Horowitz \& Whitney, 1975). These explanations will require further tests with physiological and behavioral confirmation of the possible differential effects.

In summary, the findings of the present investigation further demonstrated that sexual dimorphism in CTA extinction is not unique to rat species. On the other hand, they indicated that the phenomenon in inbred mice is strain specific at the ages tested. This finding of strain interaction might prove to be very useful for further assessment of testosterone involvement in this learning paradigm. Of course, manipulation of the hormonal variable will be required for a definitive demonstration. At this point, the strain difference offers a hypothesis of genetic involvement in producing the different levels of the hormone. Consistent with earlier reports of strain differences in rats, however, the possibility of geneenvironment interaction must also be acknowledged (Ader, 1973; Dragoin, 1971). This consideration applies equally to mouse studies, since Batty (1978a, $1978 b)$ reported that $\mathrm{DBA} / 2 \mathrm{~J}$ mice rank higher or lower than C57BL/6Fa mice in testosterone levels, depending upon the supplier and upon the sexual experience of the animal.

\section{REFERENCE NOTES}

1. Robbins, R. J. Sex-related differences in taste-aversion learning in deer mice. Paper presented at the meeting of the Psychonomic Society, Phoenix, Arizona, November 1979.

2. Ingram, D. K. Lithium chloride-induced taste aversions in $C 57 B L / 6 J$ and $D B A / 2 J$ mice. Paper presented at the meeting of the Southern Society for Philosophy and Psychology, Norfolk, Virginia, April 1979.

\section{REFERENCES}

ADER, R. 'Strain' differences in illness-induced taste aversion. Bulletin of the Psychonomic Society, 1973, 1, 253-254.

BatTy, J. Acute changes in plasma testosterone levels and their relation to measures of sexual behavior in the male house mouse (Mus musculus). Animal Behaviour, 1978, 26, 349-357. (a) 
BAtTy, J. Plasma levels of testosterone and male sexual behavior in strains of the house mouse (Mus musculus). Animal Behaviour, 1978, 26, 339-348. (b)

Belknap, J. K., Belknap, N. D., Berg, J. H., \& Coleman, R. Preabsorptive vs. postabsorptive control of ethanol intake in C57BL/6J and DBA/2J mice. Behavior Genetics, 1977, 7, 413-425.

Belknap, J. K., Coleman, R. R., \& Foster, K. Alcohol consumption and sensory threshold differences between C57BL/6J and DBA/2J mice. Physiological Psychology, 1978, 6, 71-74.

Chambers, K. C. Hormonal influences on sexual dimorphism in the rate of extinction of a conditioned taste aversion in rats. Journal of Comparative and Physiological Psychology, 1976, 90, 851-856.

Chambers, K. C., \& Sengstake, C. B. Sexually dimorphic extinction of a conditioned taste aversion in rats. Animal Learning \& Behavior, 1976, 4, 181-185.

Chambers, K. C., \& Sengstake, C. B. The pseudo-castration effects of social isolation on extinction of a taste aversion. Physiology \& Behavior, 1978, 21, 29-32.

Dragoin, W. B. Conditioning and extinction of taste aversions with variations in intensity of the CS and UCS in two strains of rats. Psychonomic Science, 1971, 22, 303-304.

Dudek, B. C., \& Fulle r, J. L. Task-dependent genetic influences on behavioral response of mice (Mus musculus) to acetaldehyde. Journal of Comparative and Physiological Psychology, 1978, 92, 749-758.

Earley, C. J., \& Leonard, B. E. Androgenic involvement in conditioned taste aversion. Hormones and Behavior, 1978, 11, $1-11$.

Eleftherjou, B. E., \& Lucas, L. A. Age-related changes in seminal vesicles and plasma testosterone levels in male mice. Gerontologia, 1974, 20, 231-238.

Horowitz, G. P., \& Whitney, G. Alcohol-induced conditioned aversion: Genotypic specificity in mice (Mus musculus). Journal of Comparative and Physiological Psychology, 1975, 89, 340-346.

Nachman, M. Learned taste and temperature aversions due to lithium chloride sickness after temporal delays. Journal of Comparative and Physiological Psychology, 1970, 73, 22-30.

RAmirez, I., \& Fuller, J. L. Genetic influence on water and sweetened water consumption in mice. Physiology \& Behavior, 1976, 16, 163-168.

Sengstake, C. B., \& Chambers, K. C. Differential effects of fluid deprivation on the acquisition and extinction phases of a conditioned taste aversion. Bulletin of the Psychonomic Society, $1979,14,85-87$.

Sengstake, C. B., Chambers, K. C., \& Thrower, J. H. The interactive effects of fluid deprivation and testosterone on the expression of a sexually dimorphic conditioned taste aversion. Journal of Comparative and Physiological Psychology, 1978, 92, 1150-1155.

Winer, B. J. Statistical principles in experimental design. New York: McGraw-Hill, 1971

(Received for publication May 16, 1980; revision accepted November 7,1980 .) 